\title{
Gefahr einer Influenza-Pandemie - sind wir vorbereitet?
}

"Eine feindliche Armee fällt in Deutschland ein, terrorisiert die Zivilbevölkerung, 20000 Kinder, Frauen und Männer werden getötet und nach wenigen Monaten ziehen die Streitkräfte wieder $\mathrm{ab}$. Ein solches Ereignis könnte mit $>\mathrm{Na}$ tionale Katastropher nur annähernd beschrieben werden. Politik und andere gesellschaftliche Kräfte würden alles tun, damit sich Vergleichbares nicht wieder ereignet. «

Diese freie Übersetzung der Einleitung eines Artikels von Farr aus dem Jahre 1855 zur Cholera-Epidemie 1848-1849 in England mag heute befremden, und dennoch beschreibt sie ein vergleichbares Ereignis: die Influenza-Epidemie 1995/96 in Deutschland. Die Übersterblichkeit lag von Dezember 1995 bis Februar 1996 (als Indikator für die Influenza-Aktivität) tatsächlich bei über 30000 Personen.

Regelmäßige Influenza-Epidemien entstehen durch die ständige Veränderung der Influenzaviren. Veränderte Oberflächenantigene führen zur Entstehung neuer Subtypen und Varianten, so daß die früher erworbene Immunität einzelner Personen, aber auch der gesamten Bevölkerung nicht mehr greift. Man nimmt an, daß die großen Pandemien dieses Jahrhunderts durch den Wirtswechsel der Viren von Wasservögeln auf Schweine und schließlich zum Menschen ausgelöst wurden.

Die letzte große Pandemie begann vor fast 30 Jahren. Millionen Erkrankte und viele Tote allein in Deutschland waren die Folge. Ob und wann eine neue Pandemie von diesen Ausmaßen zu erwarten ist, bleibt offen. Die WHO überwacht weltweit die Verbreitung der Influenza und beobachtet die Veränderun- gen der Viren. Parallel hierzu beobachten viele nationale Meldesysteme, so wie es die Arbeitsgemeinschaft Influenza (AGI) in Deutschland tut, die Auswirkungen und Verbreitungen der jährlichen »normalen« Epidemien. Anhand dieser Beobachtungen läßt sich einschätzen, wann ein Influenzavirus besonders hohe Morbiditäten oder Mortalitäten verursacht.

\section{Maßnahmen bei drohender Pandemie}

Informationen über einen neuen Influenzastamm und seine epidemiologische Bedeutung können mit modernen Kommunikationssystemen in kürzester Zeit an die Impfstoffhersteller, die Nationalen Referenzzentren (in Hannover und Berlin) und Surveillance-Systeme weitergegeben werden.

Wird mit einer Pandemie gerechnet, hat im nationalen Netzwerk das Robert Koch-Institut (als Bundesinstitut für Infektionskrankheiten) die Aufgabe, Behörden des Öffentlichen Gesundheitsdienstes, die Fachöffentlichkeit und die Bevölkerung zu informieren. Seitens der Nationalen Referenzzentren sind Empfehlungen zur Diagnostik zu erarbeiten und Testkits bereitzustellen. Das Surveillance-System verstärkt seine Beobachtungen über die Ausbreitung der Infektion.

Differenzierte Empfehlungen zum Einsatz der Schutzimpfung für die Risikogruppen und die Arbeitswelt sind entscheidend. In Deutschland fehlt es an einer Vorschrift, wonach staatliche Stellen Produktion und Verteilung von Impfstoff anordnen können. Da alle Hersteller internationale Konzerne sind, ist überdies denkbar, daß ausländische Mehrheitsgesellschafter den Export heimischer Produktion verlangen. Aber selbst, wenn die Verteilung geregelt wäre, ist eine überwiegende Impfung der Bevölkerung illusorisch, da die vorhandenen Produktionskapazitäten dem enormen kurzzeitigen Bedarf nicht nachkommen könnten. Eine erste Voraussetzung, einer Pandemie zu begegnen, wäre also die Impfung aller Personen in den Risikogruppen (ca. 20 Millionen Menschen), weil durch eine etablierte Strategie das beschriebene Defizit im Vorfeld verringert würde.

Für die Anordnung von Maßnahmen der Beobachtung, Isolierung, Einschränkung der Berufstätigkeit, Besuchsverbote für Gemeinschaftseinrichtungen und Infektionsprävention in Krankenhäusern sind die Länder zuständig. Hier kann das Robert Koch-Institut durch Empfehlungen erheblich zu einem strukturierten und einheitlichen Vorgehen in allen Bundesländern beitragen.

Da bei einer Pandemie Impfstoff kaum rechtzeitig und ausreichend verfügbar sein wird, kommt den genannten Maßnahmen (mit erheblichen Einschränkungen von Freiheitsrechten) eine große Bedeutung zu. Nur die Steigerung der Impfbeteiligung und die Akzeptanz von Grundrechtseinschränkungen könnte eine Katastrophe verhindern.
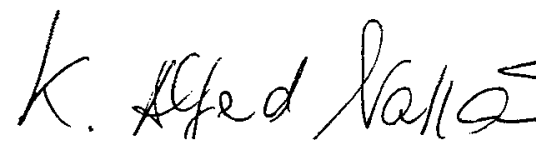

K. Alfred Nassauer

Nosokomiale Infektionen/angewandte Seuchenhygiene, Robert Koch-Institut 